\title{
Optical fibres in aeronautics, robotics and civil engineering
}

\author{
Giuseppe De Maria, Aldo Minardo, Ciro Natale, \\ Salvatore Pirozzi and Luigi Zeni \\ Dipartimento di Ingegneria dell'Informazione, Seconda Università degli Studi di Napoli \\ Aversa (CE), 81031, Italy
}

\section{Introduction}

Glass optical fibres are made from fused silica, are about the diameter of a human hair, and transmit light over large distances with very little loss. They can also be made to be sensitive to their state and environment and are therefore well suited as sensors. Optical fibres sensors (OFSs) have been the subject of a remarkable interest in the last 30 years, since they present some distinct advantages over other technologies (Culshaw \& Dakin, 1997). The principal single attractive feature of optical-fibre sensors is undoubtedly their ability to function without any interaction with electromagnetic fields. This opens applications in the electrical power industry and assists very significantly where long transmission distances of relatively weak signals are an essential part of the sensing process. The lack of electrical connections has other, broader implications. Optical sensors have major advantages when conductive fluids, such as blood or sea water, are involved. Also, the need for intrinsic safety (for example, in monitoring the presence of explosives gases or in assessing petrochemical plants) is often paramount. The optical fibre is also remarkably strong, elastic, and durable, and has found its place as an instrumentation medium for addressing smart structures, where the sensors must tolerate the environment to which the structure is subjected and therefore to be immune to large physical strain excursions, substantial temperature excursions, and often a chemically corrosive operating environment.

Fibre optic sensor technology has been a major user of technology associated with the optoelectronic and fibre optic communications industry. Many of the components associated with these industries were often developed for fibre optic sensor applications. Fibre optic sensor technology, in turn, has often been driven by the development and subsequent mass production of components to support these industries. As component prices have fallen and quality improvements have been made, the ability of fibre optic sensors to displace traditional sensors for rotation, acceleration, electric and magnetic field measurement, temperature, pressure, acoustics, vibration, linear and angular position, strain, humidity, viscosity, chemical measurements, and a host of other sensor applications has been enhanced (Udd, 2002).

In the early days of fibre optic sensor technology, most commercially successful fibre optic sensors were squarely targeted at markets where existing sensor technology was marginal 
or in many cases nonexistent. The inherent advantages of fibre optic sensors were heavily used to offset their major disadvantages of high cost and end-user unfamiliarity. The situation is changing. Laser diodes that cost \$3000 in 1979 with lifetimes measured in hours now sell for a few dollars in small quantities, have reliability of tens of thousands of hours, and are widely used in compact disc players, laser printers, laser pointers, and bar code readers. Single-mode optical fibre that cost $\$ 20 / \mathrm{m}$ in 1979 now costs less than $\$ 0.10 / \mathrm{m}$, with vastly improved optical and mechanical properties. Integrated optical devices that were not available in usable form at that time are now commonly used to support production models of fibre optic gyros. Also, they could drop in price dramatically in the future while offering ever more sophisticated optical circuits. As these trends continue, the opportunities for fibre optic sensor designers to product competitive products will increase and the technology can be expected to assume an ever more prominent position in the sensor marketplace.

In the following sections we will review the main fields in which we successfully adopted optical fibre sensor technology. In particular, we will focus on the use of distributed fibre sensors for structural health monitoring; then we will describe an application of fibre-optics Bragg grating technology in the field of aeronautic engineering. Finally, an optical fibrebased tactile sensor for robotic applications will be described. The selection of these applications has been made to clearly show how the same base device, i.e. the optical fibre, can be successfully used to address sensing problems at very different scales, from large structures down to micro-scale devices.

\section{Application in civil engineering}

The safety assessment of ordinary structures is usually based on the experimental testing of displacements or strains under the design loads, by taking into consideration measures related to a selected discrete number of points. For many engineering works of strategic significance like bridges, pipes, rising buildings, dams and tunnels, safety should be continuously assessed during their complete life-time. It is worth to note that the response of these structures, which for their nature could be damaged by severe load conditions, has to be monitored along the whole construction. When compared with traditional electrical strain gauges used for strain monitoring of large structures, OFSs have several distinguishing advantages, including:

1. A much better invulnerability to electromagnetic interference, including storms, and the potential capability of surviving in harsh environments;

2. A much less intrusive size (typically $125 \mu \mathrm{m}$ in diameter-the ideal size for embedding into composites without introducing any significant perturbation to the characteristics of the structure);

3. Greater resistance to corrosion when used in open structures, such as bridges and dams;

4. A higher temperature capacity with a widely selectable range;

5. A longer lifetime, which could probably be used throughout the working lifetime of the structure (e.g., >25 years) as optical fibres are reliable for long-term operation without degradation in performance.

Besides the above mentioned advantages, fibre optics technology offers the unique possibility to perform the experimental reading of strains over the structure in a fully distributed manner. This feature is accomplished by the so-called distributed optical fibre 
sensors, by which not only can the magnitude of a physical parameter or measurand be monitored, but also its variation along the length of the fibre can be measured. Distributed sensors are especially appropriate when there is no a priori information allowing a spatial delimitation of the sensing region. The experimental procedure consists of durable devices set on the structure at an early stage of the building process that is particularly able to detect the onset of damages or defects during the whole life-time of the structure (Measures 2002).

Truly distributed sensing techniques are commonly based on some kind of light scattering mechanism occurring inside the fibre. Spatial resolution is typically achieved by using the optical time domain reflectometry (OTDR) (Barnosky \& Jensen 1976), in which optical pulses are launched into an optical fibre and the variations in backscattering intensity caused by measurand is detected as a function of time.

While OTDR is a quite simple and established technique, it does not allow strain sensing. The latter can be achieved by exploiting the phenomenon of Brillouin scattering. In Brillouin effect an optical pump wave is scattered by acoustic waves leading to Stokes (a longer wavelength than that of the pump) or anti-Stokes (a shorter wavelength than that of the pump) components (see Fig. 1).

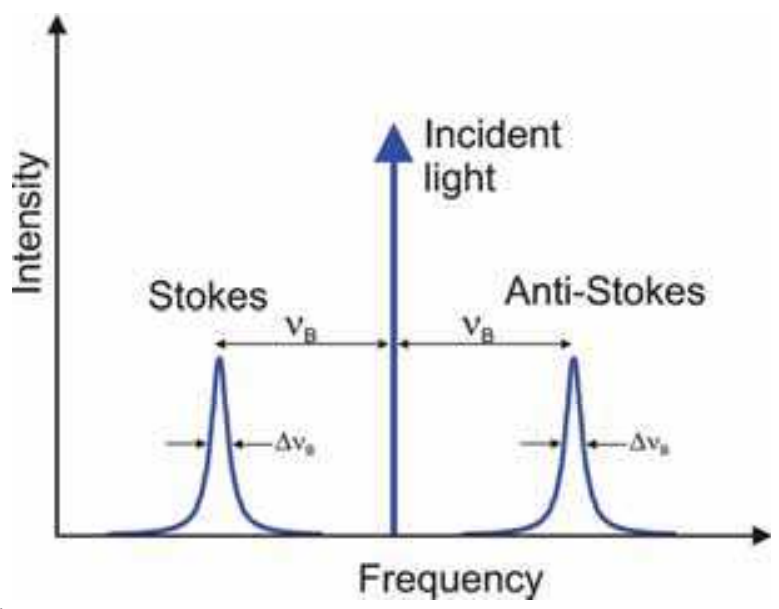

Fig. 1. The Brillouin spectrum.

Essentially, scattering occurs as a result of a Bragg-type reflection from moving diffraction gratings created from the refractive-index variations caused by acoustic waves propagating axially in the fibre material. These acoustic waves can be generated spontaneously by thermal excitation and, when this is the case, the resulting scattering effect on optical waves is known as spontaneous Brillouin scattering. However, as the optical pump power is increased the wave scattered backwards from an acoustic wave will increase in amplitude and will interfere significantly with the forward-travelling pump wave. An optical beat signal arises within the fibre, which generates a pressure wave having the same frequency as the optical beat signal, via the phenomenon of electrostriction; this pump-induced index grating scatters the pump light through Bragg diffraction. Scattered light is down-shifted or up-shifted in frequency because of the Doppler shift associated with a grating moving at the acoustic velocity $\mathrm{V}_{\mathrm{A}}$. This positive feedback, backscattering process is known as the 
stimulated Brillouin scattering (SBS). It leads to much larger backscattering at the Stokes frequency than in the spontaneous case. Brillouin effect leads to a Stokes and anti-Stokes frequency shift in the optical fibre which is given by (Agrawal 2001):

$$
v_{B}= \pm \frac{2 n V_{A}}{\lambda}
$$

where $n$ is effective refractive index of the guided mode, $V_{A}$ is the acoustic velocity and $\lambda$ is the free-space pumping wavelength. For silica fibre at a pumping wavelength of $1.55 \mu \mathrm{m}$ we have $v_{\mathrm{B}} \approx 10.8 \mathrm{GHz}$. As Brillouin frequency shift depends on both the optical refractive index and the acoustic wave velocity, it changes whenever these quantities change in response to local environmental variations and can be used to deduce the temperature and strain along the fibre. Several experiments have demonstrated an excellent linearity of the Brillouin frequency shift with respect to both fibre strain and temperature, for a wide range of these quantities. At a pump wavelength of $1.32 \mu \mathrm{m}$, a typical temperature coefficient of $1.36 \mathrm{MHz} /{ }^{\circ} \mathrm{C}$ and strain coefficient of $594.1 \mathrm{MHz} / \%$ are reported (Niklès et al., 1997).

As an example of application of distributed sensors in the field of structural health monitoring (SHM), we report the results of strain measurements carried out along an 8meters-long "I" steel beam subjected to load (Bernini et al., 2006a). In particular, the measurements were carried out by using a transportable prototype able to carry out SBS distributed sensing in the time-domain. Two tests were performed: the first one refers to the integral beam, while the second one was performed after the intentional formation of a defect localized over a 10cm-long portion of the beam. The results, shown in Figs. 2 and 3, demonstrated the capability of the sensor to identify both position and amount of damage.

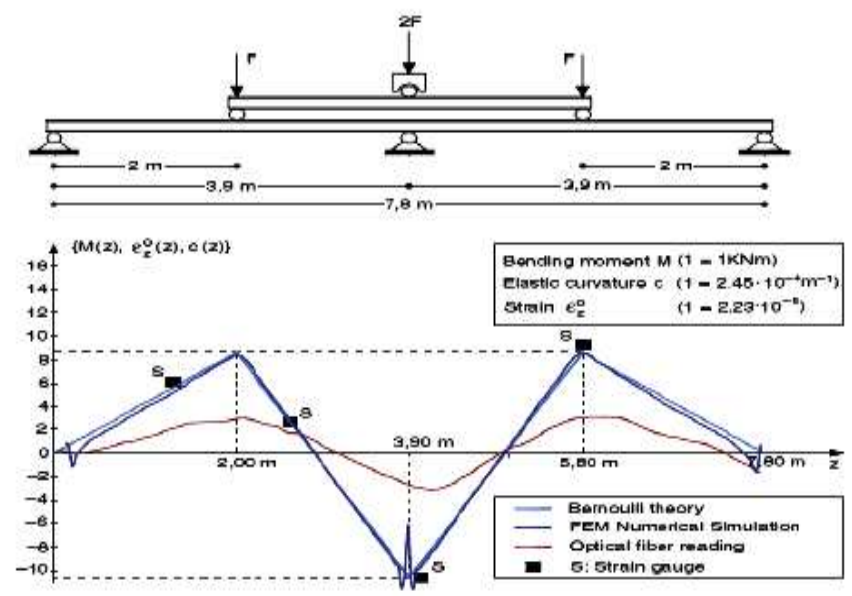

Fig. 2. Strain profile measured along the integral beam and comparison with the theoretical profile. 


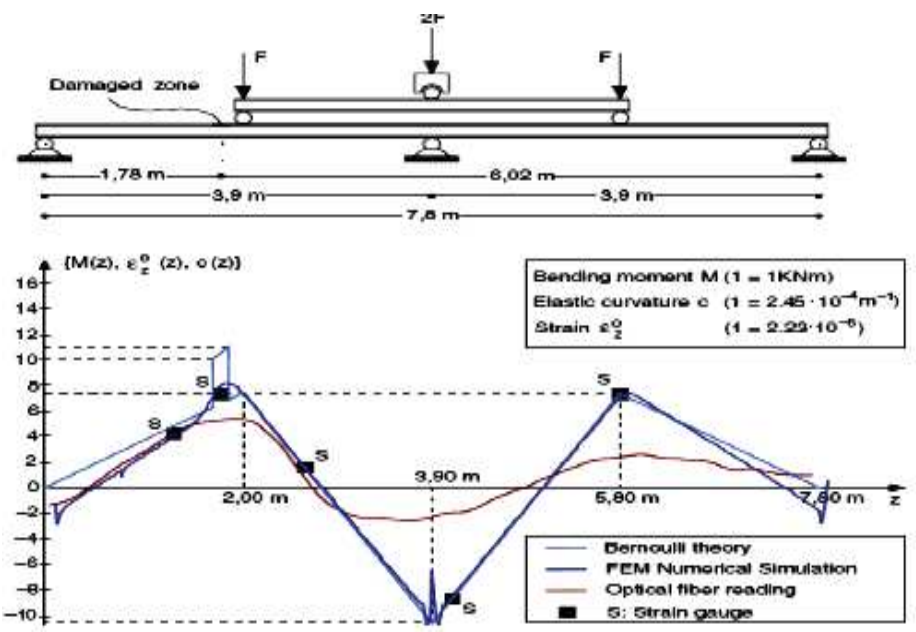

Fig. 3. Strain profile measured along the beam with a defect and comparison with the theoretical profile.

Distributed strain measurements can be carried out also by using a detection scheme operating in the frequency-domain (Garus et al., 1996). In this case, the pump beam intensity is sinusoidally-modulated, and the corresponding modulation induced on the $\mathrm{cw}$ probe beam intensity is coherently measured in magnitude and phase. Synchronous detection offered by frequency-domain schemes allows for spatial resolution and accuracy typically higher than time-domain approaches (Bernini et al., 2002). As an example, we report strain measurements carried out along a $4 \mathrm{~m}$-long, L-shaped aluminium beam subjected to a $2 \mathrm{~kg}$ load at the beam middle section (Bernini et al. 2006b).

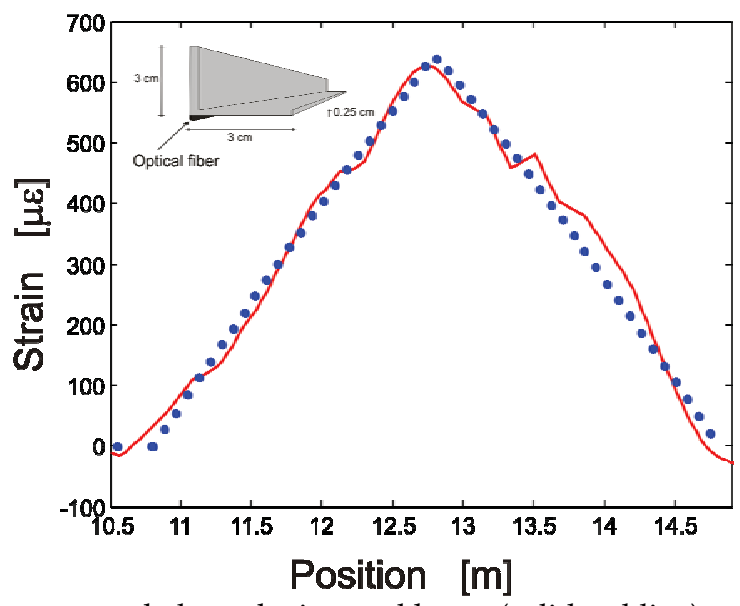

Fig. 4. Strain profile measured along the integral beam (solid red line), and comparison with the theoretical profile (circles). The inset shows the optical fibre position with respect to the loaded beam. 
The comparison between the measurement and the strain profile calculated by a finiteelements method (FEM), shown in Fig. 4, demonstrates the high level of accuracy offered by frequency-domain SBS approaches.

Another interesting application of fibre-optic distributed sensor, consists in the monitoring of pipelines. In particular, it has been demonstrated that by measuring the strain profile along three longitudinal directions of a pipe, it is possible to identify the position and the vectorial amount of pipe dislocation (Bernini et al., 2008). Such a technique can be applied, both directly for pipeline integrity monitoring, both in the geotechnical field for the monitoring of deformation of the soil surrounding the pipe itself. Experimental tests were performed by using a frequency-domain SBS-based sensor. In particular, a $1.5 \mathrm{~m}$-long polyethylene (PE) pipe was used, along which strains were read by attaching an optical fibre running along three longitudinal directions angularly spaced of $120^{\circ}$. As an example, we show in Figure 5 the measurements carried out after displacing a $25 \mathrm{~cm}$-long section of the pipe of $20 \mathrm{~cm}$ along the $-x$ direction.

An appealing aspect of such measurements is the possibility to reconstruct, section by section, the spatial deformation of the pipe. Actually, by making simple calculations based on the Bernoulli theory, pipe dislocation along the $x$ - and $y$ - directions can be deduced at each pipe section. A 3D reconstruction of the deformed pipe, obtained by opportune processing of the data shown in Fig. 5, is reported in Fig. 6, along with the ideal reconstruction obtained by using the numerically calculated deformations.

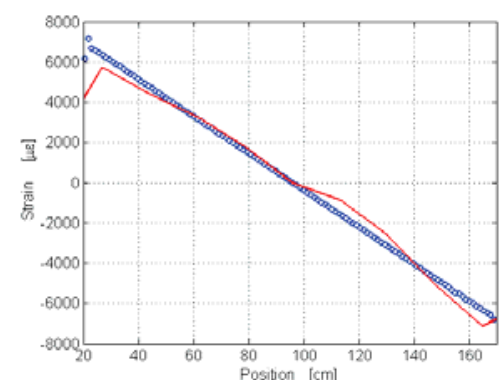

(a)

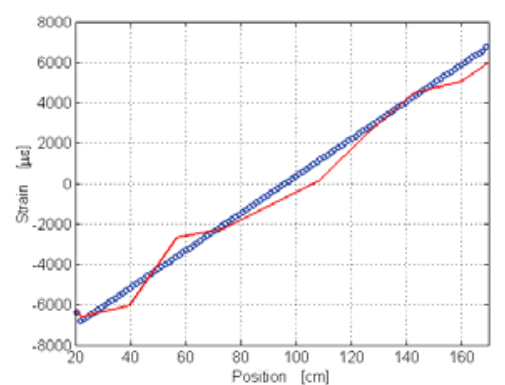

(b)

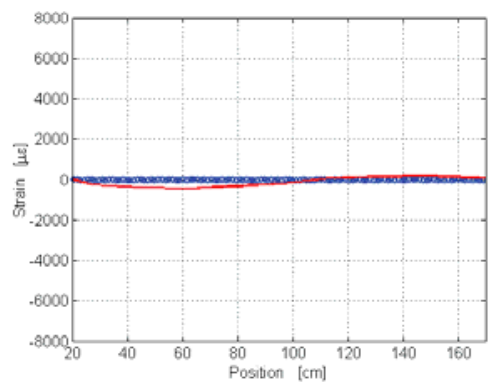

(c)

Fig. 5. Experimental (solid red line) and numerically calculated (circles) strain profiles along fibre A (a), fibre B (b) and fibre C (c), for a $20-\mathrm{cm}$ displacement of the pipeline along the negative $x$-direction. 


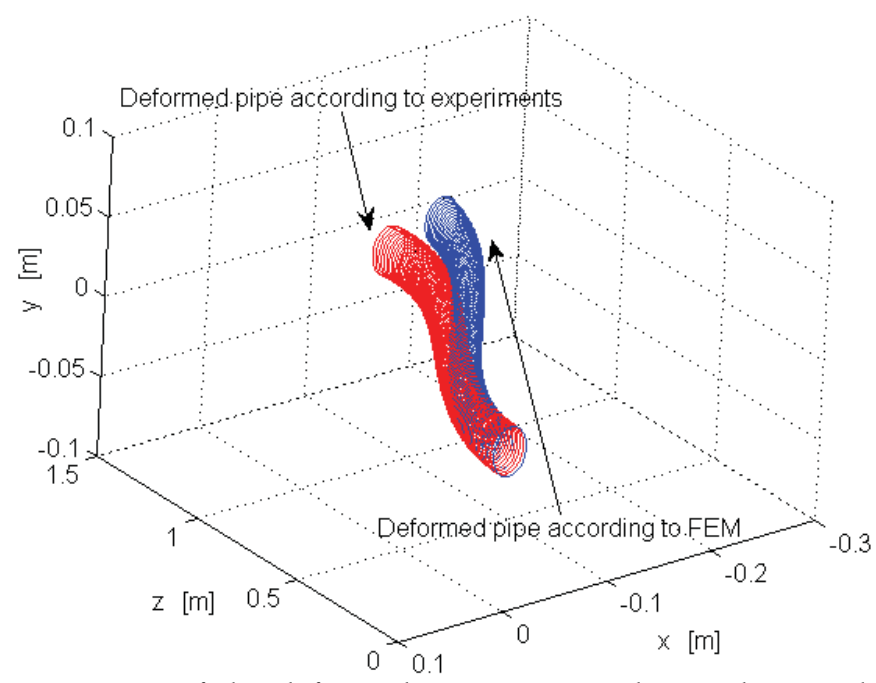

Fig. 6. 3D representation of the deformed pipe, estimated according to the experimental strains (leftmost red pipe) and the FEM numerically calculated strains (rightmost blue pipe).

\section{Application in aeronautics}

Many research projects worldwide tackled the problem of noise and vibration reduction in aeronautic structures for improvement of both cabin comfort and of structural health. Among these, the European project MESEMA had the main objective of designing and implementing an active noise control system on a full-scale test rig consisting of a segment of a civil aircraft fuselage. The problem addressed was the reduction of cabin noise in a broad frequency band, ranging from $100 \mathrm{~Hz}$ to $500 \mathrm{~Hz}$. The active control was realized by using actuators based on a proven and patented concept, the magnetostrictive auxiliary mass damper, which was optimally designed for this application (May et al. 2006).
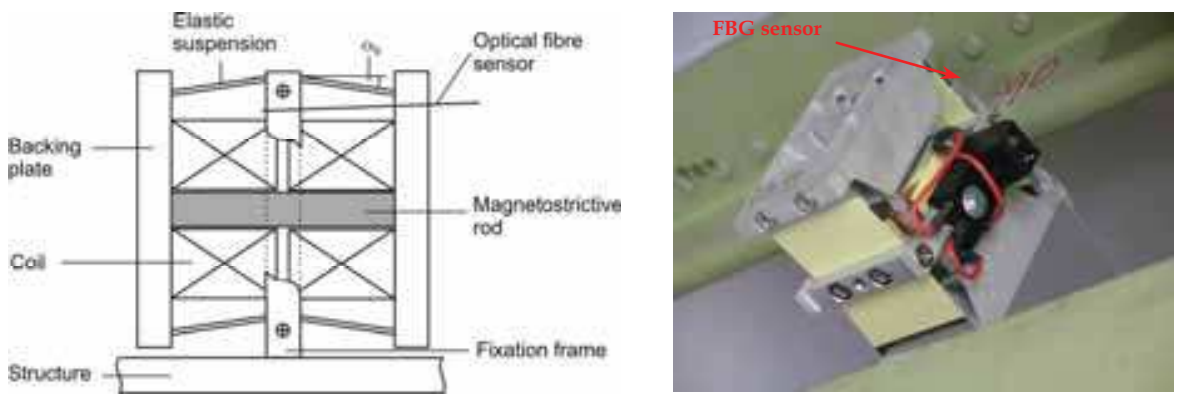

Fig. 7. Actuator with integrated optical sensor: schematic and actual installation on the aeronautic structure.

Unfortunately, the dynamic behaviour of this device was affected by two sources of nonlinearity. The first one due to the elastic suspension kinematics used for displacement 
amplification; the second one due to the hysteretic behaviour of the magnetostrictive material. In order to overcome the limitation and negative effects of these nonlinearities within the main control system, the actuator was equipped with an optical sensor based on a Fibre Bragg Grating (FBG) used for low-level actuator control to measure the displacement of the inertial mass, so as to estimate the state of the mechanical system.

The possibility to use an FBG as strain sensor is related to the changes in its optical reflection spectrum produced by an applied strain (Kersey et al. 1997). In particular, the centre wavelength of the FBG reflection spectrum is linearly dependent on strain, so that the latter can be retrieved on a wavelength-encoding basis. In case of static strain measurements, FBG interrogation is typically performed by using a wideband optical source, such as an LED, while monitoring the portion of the source spectrum reflected by the grating. By doing so, the centre wavelength of the reflected spectrum provides a direct measurement of the applied strain. Such an approach typically involves the use of an optical spectrum analyzer in order to acquire the reflection spectrum, so that it is inherently slow and not suitable for dynamic measurements. For our dynamic measurements, we choose to adopt a narrowband demodulation approach, capable of detecting strain changes up to the $\mathrm{kHz}$ range. Apart from the possibility to detect fast strain changes, the narrowband demodulation technique presents other advantages, such as high signal-to-noise ratio, low cost, and ease of use (Zhao \& Liao 2004). The principle of operation of the narrowband technique can be understood by looking at the optical set-up connected to the FBG, and schematically illustrated in Fig. 8. Light emitted by a distributed feedback (DBF) diode laser is sent to a Y-coupler, which directs laser light to the FBG. Light reflected from the FBG is then re-directed to a highspeed photodiode (PD). Assuming that the laser frequency is within the linear range of the FBG reflection slope, the strain signal will produce a change in the reflected optical power, which is measured by the photodetector. In other words, using a narrowband laser at a fixed wavelength permits to convert any FBG spectrum wavelength modulation induced by the strain signal, into an intensity signal, which is finally converted into an electrical signal by the photodiode. In our measuring set-up, the electrical output from the photodiode was sent to a conditioning electronics, which basically comprises a bandpass filter used to eliminate the dc component and the high-frequency noise.
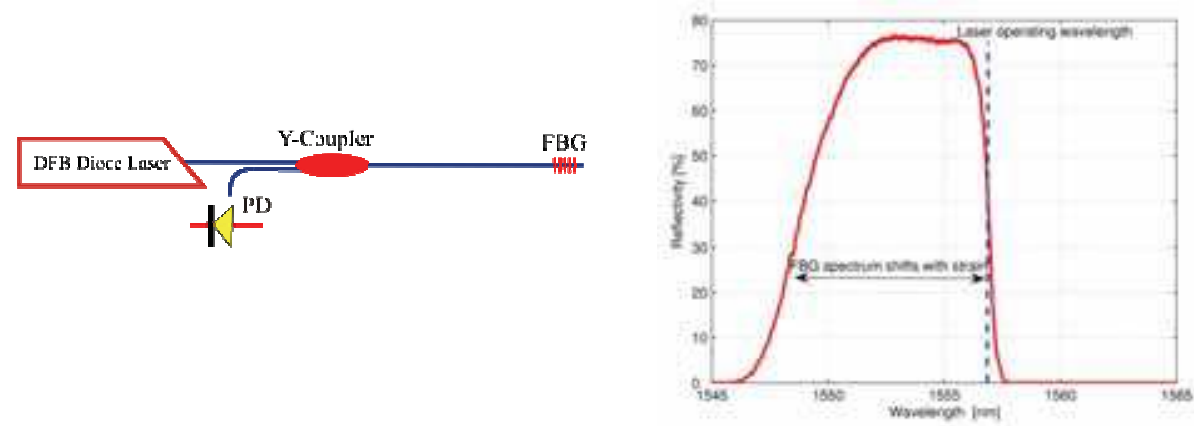

Fig. 8. FBG interrogation scheme (left) and reflectivity spectrum of the grating (right).

Figure 8 shows the reflectivity spectrum of the FBG used in our experiments, as measured at room temperature and with no applied strain. It can be seen that the spectrum has a quasi- 
flat top from $1552 \mathrm{~nm}$ to $1556 \mathrm{~nm}$, with a peak reflectivity greater than 75\%. The leading edge of the FBG reflection curve extends from $1547.28 \mathrm{~nm}$ to $1550.95 \mathrm{~nm}$ (measured from $10 \%$ to $90 \%$ of maximum reflectivity), whereas the trailing edge extends from $1556.37 \mathrm{~nm}$ to $1557.24 \mathrm{~nm}$. The trailing edge of the FBG reflection curve allowed for about $72 \mathrm{GHz}$ linear slope width, and it was chosen as the operating range due to the higher linearity exhibited by the FBG reflectivity in this spectral portion. In order to keep a linear relationship between the reflected optical power and the strain signal, the DFB laser emitting wavelength must lie within the trailing edge of the FBG reflectivity spectrum (see Fig. 8). Moreover, assuming a typical FBG curve shift of $1 \mathrm{~nm}$ for an applied strain of $1000 \mu \varepsilon$ ((Kersey et al. 1997), the strain level must be kept lower than $870 \mu \varepsilon$ in order to avoid sensor output saturation.

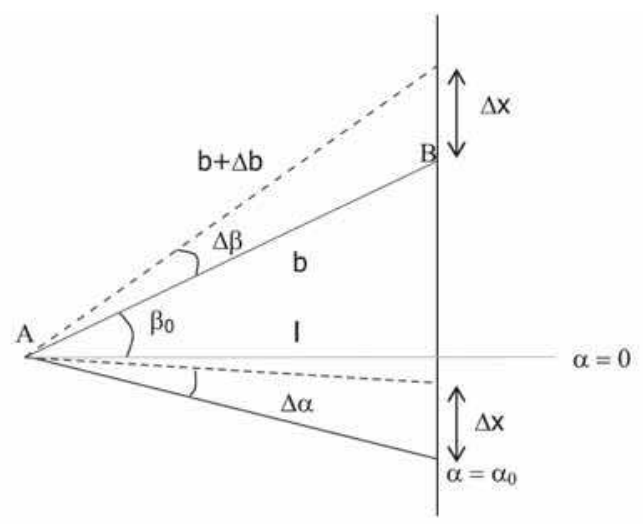

Fig. 9. Sketch of sensor mounting setup.

Before attaching the FBG to the magnetostrictive actuator, the portion of fibre jacket corresponding to the grating position was removed, so as to avoid strain transfer loss from actuator to FBG. The FBG was then pre-stressed and glued to the actuator by epoxy resin. Pre-stressing of the FBG was necessary in order to avoid buckling of the fibre in which the sensor is written. Mounting of the FBG was carried out, such that the output of the optical sensor is a measurement of one of the state variables of the system, $\alpha(t)$. A sketch of the mounting set-up is shown in Fig. 9. As the output provided by the FBG is proportional to the strain of the fibre segment between the two bonding points A and B, the relationship between the strain and the angular displacement has to be determined. Such a relationship can be obtained by means of geometrical considerations. Referring to Fig. 9, let us start by considering that, under real experimental conditions, the displacement $\Delta \mathrm{x}$ of the moving mass is much smaller than the lever arm length 1 , hence $\Delta \mathrm{x} \approx l \Delta \alpha$. Moreover, $\Delta \mathrm{x}$ is also much smaller than the fibre segment $b$, so that we can also assume $\Delta \beta \approx 0$. Under this approximation, the displacement $\Delta \mathrm{x}$ is nearly equal to $\Delta \mathrm{b}$. As the optical sensor provides an output proportional to the strain $\Delta \mathrm{b} / \mathrm{b}$, we can finally write:

$$
\epsilon \equiv \frac{\Delta b}{b} \approx \frac{l}{b} \Delta \alpha
$$

Hence, under the small signal hypothesis, the quantity measured by the FBG is directly proportional to the angle variation $\Delta \alpha=\alpha-\alpha 0$. After bonding the FBG, the DFB laser emitting 
wavelength was temperature-tuned in order to guarantee a linear response of the sensor, over the whole range of actuator displacements. Calibration of the FBG was carried out by aid of an accelerometer mounted to the moving mass, so as to measure the acceleration along the vertical axis. The sensor sensitivity was estimated by comparing the measured frequency response functions from the excitation signal to the second time derivative of the FBG output signal, and from the excitation signal to the accelerometer output signal, respectively. Results are shown in Fig. 10. Based on the calibration constant of the accelerometer, we estimated an FBG sensitivity of about $37.6 \mathrm{mV} / \mu \mathrm{m}$.
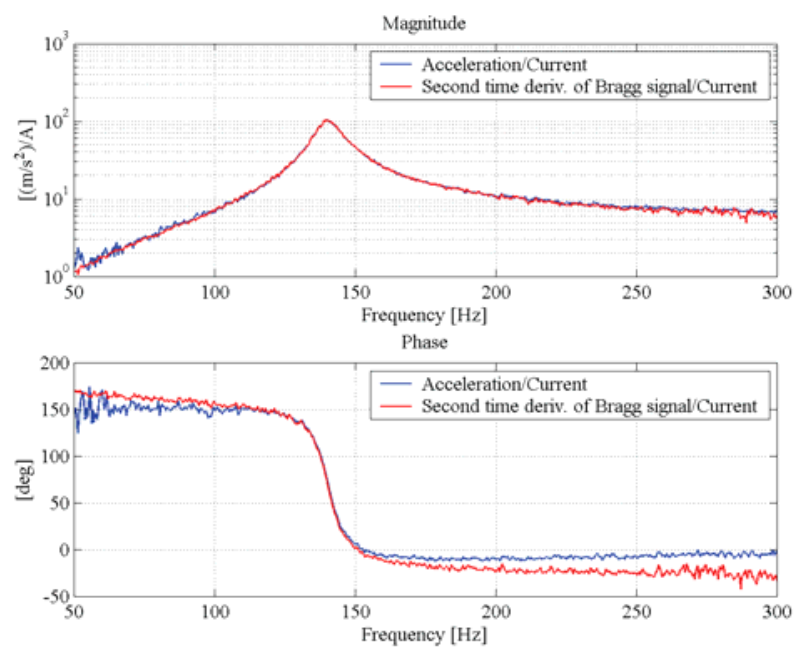

Fig. 10. Bragg calibration result: accelerometer (blue), Bragg second time derivative (red).

As mentioned before, the actuator nonlinearity causes a dependence of the structural frequency response on the amplitude of the driving current. This nonlinearity affects the satisfaction of the force requirements and makes the actuator difficult to implement in the noise control system. Therefore, a feedback controller exploiting the FBG measurement has been designed to reduce alterations of the structural response due to variations of the input current level. The control scheme is reported in Fig. 11 and the details of the design procedure can be found in (Cavallo et al. 2009). The control law basically tries to impose a desired dynamic behaviour to the actuator as specified in the reference model, which is linear time-invariant system with a fixed resonant frequency, so that the actual resonant frequency does not depend on the current level any more. 


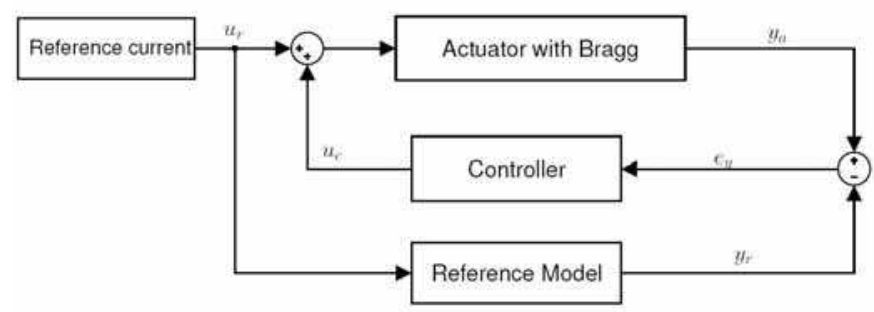

Fig. 11. Actuator feedback control scheme.
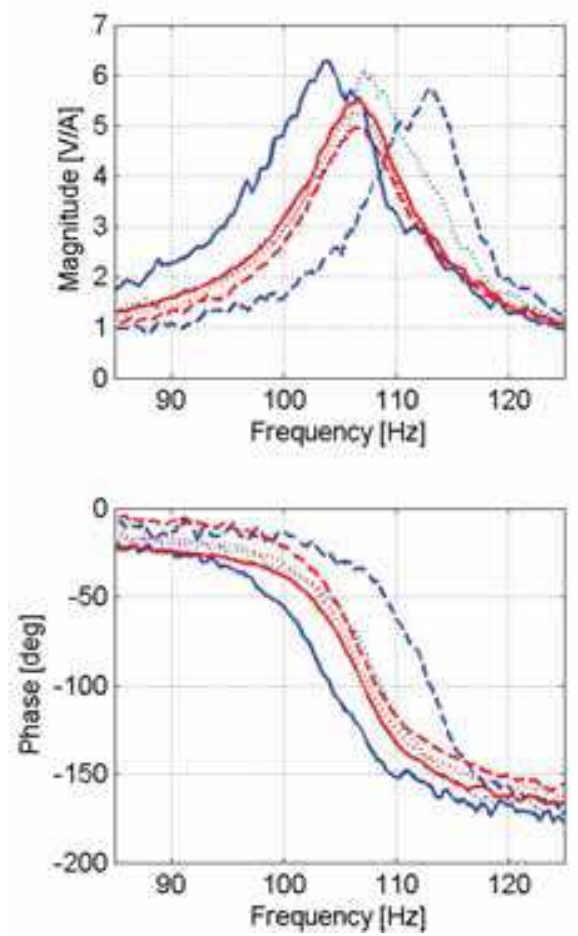

Fig. 12. Actuator behaviour at different current levels: control off (blue), control on (red).

Fig. 12 reports the experimental results obtained applying this control scheme. The blue lines represent the actuator frequency response function measured at three different input currents without the feedback control. In the same figure, it is possible to see the positive effects on the actuator behaviour of the feedback control exploiting the sensor measurement. In fact, the red lines represent the same FRFs measured with the control loop activated, and they all exhibit the same resonant frequency, resulting in a phase shift almost insensitive to the input current amplitude. Of course such a characteristic is mandatory when the actuator resonance is exploited to actively control the vibrations of a flexible structure subject to a disturbance force field in a frequency range containing the actuator resonant frequency itself. 


\section{Application in robotics}

The number of application domains of robotic systems is rapidly growing and in particular the service robotics is becoming the most popular. In such application field a high degree of autonomy is required for the robot and thus a large number of exteroceptive sensors appear necessary. When multifingered robotic hands are considered, the requirement of minimally invasiveness for the sensory system is of major importance due to the limited space available in a mechanical structure with several degrees of freedom. In a robotic hand, different exteroceptive sensors are required to ensure stable grasping and manipulation of objects. Among these, sensing of both contact point and contact force appears mandatory for any control algorithm which intends to achieve such goals. Even though many different technologies have been explored and tested to build tactile sensors, like piezo-resistive (Liu et al., 1993), capacitive (Morimura et al., 2000), piezoelectric (Krishna \& Rajanna, 2002), magneto-resistive (Tanie, 1986), optoelectronic approaches demonstrated their potential since the beginning of tactile sensors development (Maekawa et al., 1993). Also, on the market optoelectronic tactile sensors can be found that measure distributed tactile information, but such tactile information is generally limited to pressure force, and spatial resolution is coarse, a few millimetres order. Generally, a commercial sensor accurately responds to a load of $0.25 \mathrm{~N}$ or more up to $2 \mathrm{~N}$, but such a range can be too narrow for manipulation tasks. More recently, a number of different optical approaches have been pursued, among which the solution based on an LEDs matrix has been presented in (Rossiter \& Mukai, 2005) and the solution based on a CCD camera is reported in (Ohka et al., 2006).

Among optical approaches, those based on the use of optical fibres appear particularly suitable for pressure sensing, thanks to the low size and minimum invasivity of fibres themselves. Since the advent of fibre optics, it has been recognized that optical fibres can be used as effective pressure (and tactile) sensors. One of the earliest demonstrations of such a capability relied on the pressure-induced displacement of a diaphragm placed close to the tip of an optical fibre (Cook \& Hamm, 1979). The fibre was operated in reflection mode, so that changes in reflected intensity can be used as a measure of the pressure applied on the diaphragm. In case of tactile sensing, such an approach presents the disadvantage of requiring a complex micromachining at the tip of the fibre. Another possible approach is based on the intensity loss resulting from pressure-induced bending of the fibre (Fields et al., 1980). However, in this case the response of the sensor is highly nonlinear due to the exponential dependence of the bending loss on the radius of curvature of the fibre. More complex examples can be found based on interferometric approaches, where the changes in the optical phase are used as transducer mechanism to sense the pressure (Saran et al., 2006, Wang et al., 2001, Yuan et al., 2005). Interferometric sensors exhibit high sensitivity, but also present some disadvantages, such as low tolerance to external disturbances, and periodicity in their response. 

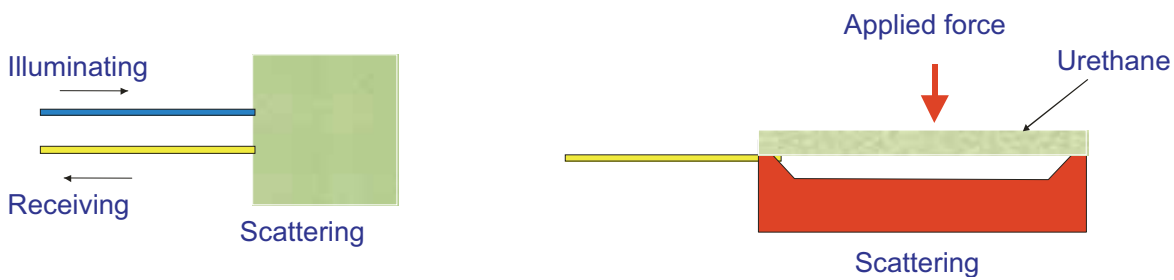

Fig. 13. Sketch of the single sensing element (taxel).

Recently, we proposed a solution based on the scattering of the light illuminating the surface of urethane foam (De Maria et al., 2008). The configuration makes use of a couple of emitter/receiver fibres placed at the edge of a micromachined well covered by the foam. The distance between the two fibres can be chosen in order to ensure a desired sensitivity of the sensing element. As a demonstration of the effectiveness of the proposed configuration, we present the results of two sensors, in which the relative distance between the two fibres was properly selected in order to fit the range of pressures to be detected. Top and lateral schematic views of a single taxel are shown in Fig. 13. The sensor works as follows: the light emitted by the illuminating fibre is scattered by the internal surface of the urethane foam and a fraction of its power is collected by the receiving fibre, depending on the applied pressure. In particular, when one applies a pressure on the external surface of the urethane foam, the distance between the tip of the collecting fibre and the internal surface of the foam is reduced, and this will result in an increased fraction of power collected by the receiving fibre. The use of a scattering surface, such as that of the urethane foam employed for the realization of the prototypes, is justified by the fact the multiple scattering permits to smooth (average-out) local variation of light intensity within the cavity, and thus reduce the sensitivity of the collected power on micro-displacements of the illuminating and/or receiving fibre. As the power collected by the receiving fibre is a function of the pressure applied on the foam surface, it can be used as a measure of the applied force. Obviously, the collected light is also a function of the relative distance between the illuminating and the receiving fibres. In our experiments, such a distance was kept constant and was not a function of the applied force. However, we can exploit such dependence, by choosing an opportune distance giving rise to a desired sensitivity of the sensor on the applied pressure. Generally speaking, a smaller distance will result in a higher sensitivity, so that smaller pressures can be measured.

On the other hand, a higher sensitivity implies a reduced dynamic range, i.e. the sensor response will saturate at lower pressure levels. Hence, a trade-off must be found between sensitivity and dynamic range.

One advantage of the proposed technique is that it can be easily extended to a number of taxels, so as to acquire a pressure distribution. Figure 14 shows a possible configuration of a matrix of taxels to realize a complete tactile sensor able to detect both contact point and contact force applied on a finite area.

Two different taxels have been produced with the same well and two different distances between the emitting/receiving fibres, i.e. $10 \mu \mathrm{m}$ and $200 \mu \mathrm{m}$. The micromachined well size is $5 \times 5 \mathrm{~mm}^{2}$. The optical source was a superluminescent LED operating at a central wavelength of $1550 \mathrm{~nm}$, and having an output optical power of $3 \mathrm{~mW}$. The output pigtail of 
the source was connected to the illuminating fibre, whereas the receiving fibre was connected to an InGaAs photodiode, whose output signal was fed to an oscilloscope having an input impedance of $1 \mathrm{M} \Omega$. Both illuminating and receiving fibres were SMF-28, singlemode optical fibres. The two prototypes have been calibrated with a load cell mounted as shown in Fig. 15. The results corresponding to the calibration of the first prototype are reported in Fig. 16 (left), where the output voltage, proportional to the optical power collected by the receiving fibre, is plotted against the load applied to the sensor. As expected, the sensitivity is very high but with a limited dynamic range. Moreover, to test the repeatability of the measurements, different sets of measurements have been collected and two of them are reported in the figure.

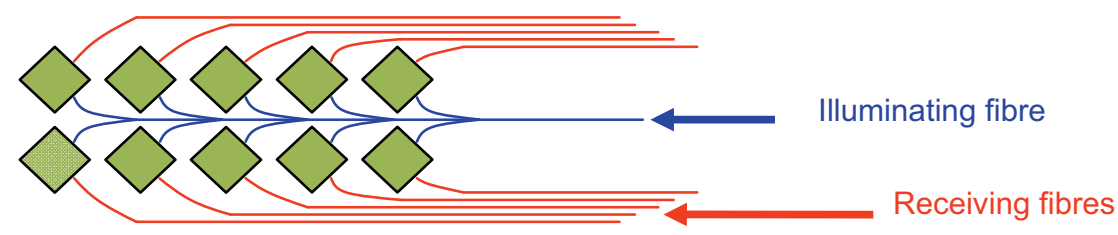

Fig. 14. Schematic diagram of a 10-taxel tactile sensor.

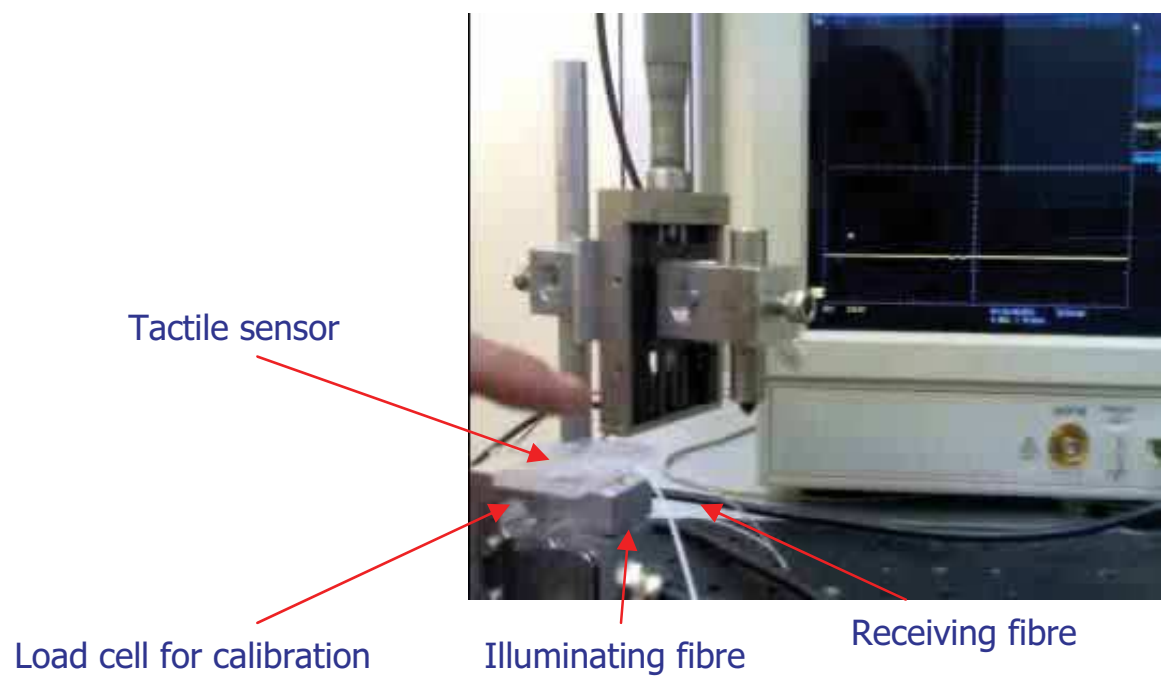

Fig. 15. Experimental set-up for the fibre-optics based taxel.

The second prototype, as expected, had a lower sensitivity but wider dynamic range, as shown by the calibration curve of Fig. 16 (right). In both cases, the sensitivity is certainly better than the typical values of commercial optical tactile sensors. 


\section{Conclusions}

In this chapter, a number of experimental demonstrations on the use of the optical fibre sensor technology have been reported. It has been shown that different application fields can take advantage of the peculiar characteristics of optical fibre sensors. In particular, distributed fibre sensors have great potentiality in the field of structural health monitoring, as they permit to perform continuous measurements of the quantity of interest. On the other hand, fibre Bragg grating technology offers high sensitivity and accuracy, and in general it benefits from the immunity to electromagnetic interference, in common with other fibreoptic sensors. Finally, the small size and minimally invasiveness of optical fibres have been demonstrated to be useful in robotic applications, where the use of fibre-optics may lead to efficient exteroceptive sensing systems.
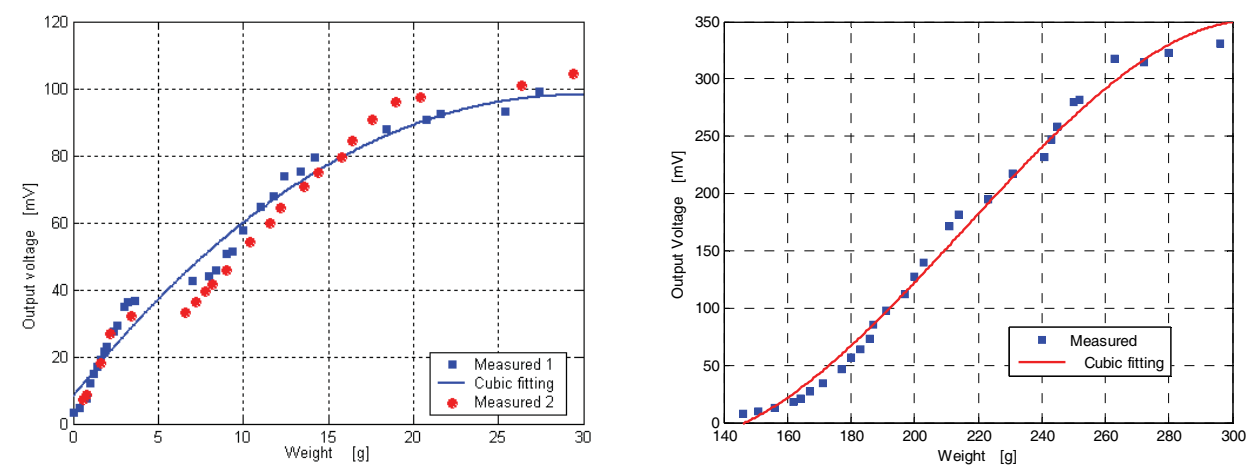

Fig. 16. Calibration curves of the first prototype with $10 \mu \mathrm{m}$ distance between the fibres (left) and of the second prototype with $200 \mu \mathrm{m}$ distance between the fibres (right).

\section{References}

Agrawal, G.P. (2001). Nonlinear Fibre Optics. Academic Press, San Diego.

Barnoski, J.K. \& Jensen, S.M. (1976). Fibre waveguides: A novel technique for investigating attenuation characteristics. Appl. Opt. Vol. 15, No. 9, 2112-2115.

Bernini, R.; Crocco, L.; Minardo, A.; Soldovieri, F. \& Zeni, L. (2002). Frequency-domain approach to distributed fibre-optic Brillouin sensing. Opt. Lett. Vol. 27, No. 5, 288290.

Bernini, R.; Fraldi, M.; Minardo, A.; Minatolo, V.; Carannante, F.; Nunziante, L. \& Zeni, L. (2006a). Identification of defects and strain error estimation for bending steel beams using time domain Brillouin distributed fibre sensors. Smart Materials and Structures Vol. 2, 612-622.

Bernini, R.; Minardo, A. \& Zeni, L. (2006b). An accurate high resolution technique for distributed sensing based on frequency domain Brillouin scattering. IEEE Photonics Technology Letters Vol. 18, No. 1, 280-282. 
Bernini, R.; Minardo, A.\& Zeni, L. (2008). Vectorial dislocation monitoring of pipelines by use of Brillouin-based fibre-optics sensors. Smart Materials and. Structures. Vol. 17, 015006.

Cavallo, A.; May, C.; Minardo, A.; Natale, C.; Pagliarulo, P. \& Pirozzi, P. (2009). Modelling and control of a smart auxiliary mass damper equipped with a Bragg grating for active vibration control, Sensors and Actuators A, in press.

Cook, R.O. \& Hamm, C.W. (1979). Fibre optic lever displacement transducer. Appl. Opt. Vol. 18, No. 19, 3230-3241.

Culshaw, B. \& Dakin, J. 1997. Optical Fibre sensors Vol. 4. Artech House Publishers, 0890069409.

De Maria, G.; Minardo, A.; Natale, C.; Pirozzi, S. \& Zeni, L. (2008). Optoelectronic Tactile Sensor Based on Micromachined Scattering Wells. FIRST MEDITERRANEAN PHOTONICS CONFERENCE, European Optical Society Topical Meeting, 25-28 June 2008, Ischia, Italy.

Fields, J.N.; Asawa, C.K.; Ramer, O.G. \& Barnowski, M.K. (1980). Fibre Optic Pressure Sensor. J. Acoust. Soc. Am., Vol. 67, 816-818.

Garus, D.; Krebber, K.; Schliep, F. \& Gogolla, T. (1996). Distributed sensing technique based on Brillouin optical-fibre frequency-domain analysis. Opt. Lett., Vol. 21, No. 17, 1402-1404.

Kersey, A. D.; Davis, M. A.; Patrick, H. J.; LeBlanc, M.; Poo, K. P.; Askins, A.G.; Putnam, M. A. \& Friebele, E. J. (1997). Fibre grating sensors, Journal of Lightw. Technol., vol. 15, no. 8, pp. 1442-1462.

Krishna, G.M. \& Rajanna, K. (2002). Tactile sensor based on piezoelectric resonance. Proc. of 2002 IEEE Conference on Sensor, pp. 1643- 1647.

Liu, L.; Zheng, X. \& Li, Z. (1993). An array tactile sensor with piezoresistive with single crystal silicon diaphragm. Sensors and Actuators-A32, 193-196.

Maekawa, H.; Tanie, K. \& Komoriya, K. (1993). A finger-shaped tactile sensor using an optical waveguide. Proc. of 1993 IEEE International Conference on Systems, Man and Cybernetics, pp. 403-408.

Measures, R.M. (2002). Structural monitoring with fibre optic technology. Academic press, San Diego.

Morimura, H.; Shigematsu, S. \& Machinda, K. (2000). A novel sensor cell architecture and sensing circuit scheme for capacitive fingerprint sensors. IEEE Journal of Solid State Circuits, Vol, 35, 724-731.

May, C.; Pagliarulo, P. \& Janocha, H. (2006). Optimisation of a magnetostrictive auxiliary mass damper. Proc. 10th International Conference on New Actuators ACTUATOR2006, Bremen, Germany, pp. 344-348.

Nikles, M.; Thevenaz, L. \& Robert, P.A. 1997. Brillouin gain spectrum characterization in single-mode optical fibres. J. Lightw. Technol., Vol. 15, No. 10, 1842 - 1851.

Ohka, M.; Kobayashi, H.; Takata, J. \& Mitsuya, Y. (2006). Sensing Precision of an Optical Three-axis Tactile Sensor for a Robotic Finger. Proc. Of the 15th IEEE International Symposium on Robot and Human Interactive Communication, pp. 214-219.

Rossiter, J. \& Mukai, T. (2005). A novel tactile sensor using a matrix of LEDs operating in both photoemitter and photodetector modes. Proc. of 2005 IEEE Conference on Sensor, pp. 994-997. 
Saran, A.; Abeysinghe, D.C. \& Boyd, J.T. (2006. Microelectromechanical system pressure sensor integrated onto optical fibre by anodic bonding. Appl. Opt., Vol. 45, 17371742.

Tanie, K. (1986). Advances in tactile sensors for robotics. Proc. of the 6th Sensor Symposium Japan, pp. 63-68.

Udd. E. (2002). Overview of fibre optic sensors, In: Fibre Optic Sensors. Francis T. S. Yu; Shizhuo Yin, pp. 1-40, Routledge, 978-0-203-90946-1, USA.

Wang, A.; Xiao, H.; Wang, J.; Wang, Z.; Zhao, W. \& May, R.G. (2001). Self-calibrated interferometric-based-optical fibre sensors. J. Lightw. Technol., Vol. 19, No. 10, 14951501.

Yuan, S.; Ansari, F.; Liu, X. \& Zhao, Y. (2005). Optical fibre based dynamic pressure sensor for WIM sensor. Sens. and Actuat. A, Vol. 120, No. 1, 53-58.

Zhao, Y. \& Liao, Y. (2004) "Discrimination methods and demodulation techniques for fibre Bragg grating sensors", Opt. Lasers Eng., vol. 41, pp. 1-18. 


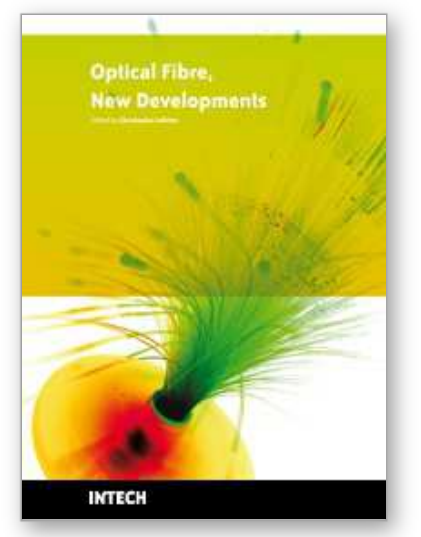

\section{Optical Fiber New Developments}

Edited by Christophe Lethien

ISBN 978-953-7619-50-3

Hard cover, 586 pages

Publisher InTech

Published online 01, December, 2009

Published in print edition December, 2009

The optical fibre technology is one of the hop topics developed in the beginning of the 21 th century and could substantially benefit applications dealing with lighting, sensing and communication systems. Many improvements have been made in the past years to reduce the fibre attenuation and to improve the fibre performance. Nowadays, new applications have been developed over the scientific community and this book fits this paradigm. It summarizes the current status of know-how in optical fibre applications and represents a further source of information dealing with two main topics: the development of fibre optics sensors, and the application of optical fibre for telecommunication systems.

\section{How to reference}

In order to correctly reference this scholarly work, feel free to copy and paste the following:

Giuseppe De Maria, Aldo Minardo, Ciro Natale, Salvatore Pirozzi and Luigi Zeni (2009). Optical Fibres in Aeronautics, Robotics and Civil Engineering, Optical Fiber New Developments, Christophe Lethien (Ed.), ISBN: 978-953-7619-50-3, InTech, Available from: http://www.intechopen.com/books/optical-fiber-newdevelopments/optical-fibres-in-aeronautics-robotics-and-civil-engineering

\section{INTECH}

open science | open minds

\section{InTech Europe}

University Campus STeP Ri

Slavka Krautzeka 83/A

51000 Rijeka, Croatia

Phone: +385 (51) 770447

Fax: +385 (51) 686166

www.intechopen.com

\section{InTech China}

Unit 405, Office Block, Hotel Equatorial Shanghai

No.65, Yan An Road (West), Shanghai, 200040, China

中国上海市延安西路65号上海国际贵都大饭店办公楼 405 单元

Phone: +86-21-62489820

Fax: $+86-21-62489821$ 
(C) 2009 The Author(s). Licensee IntechOpen. This chapter is distributed under the terms of the Creative Commons Attribution-NonCommercial-ShareAlike-3.0 License, which permits use, distribution and reproduction for non-commercial purposes, provided the original is properly cited and derivative works building on this content are distributed under the same license. 